livraisons

d'Histoire

de l'Architecture

\section{Livraisons de l'histoire de l'architecture}

24 | 2012

Le phare et l'architecte

\title{
Louis Quételart et le phare de La Canche au Touquet
}

Louis Quételart's «Phare de la Canche » at Le Touquet

Louis Quételart und der Leuchtturm an der Mündung der Canche in Le Touquet

\section{Anne Lefebvre et Olivier Liardet}

\section{OpenEdition}

Journals

Édition électronique

URL : http://journals.openedition.org/lha/103

DOI : $10.4000 /$ /ha. 103

ISSN : 1960-5994

Éditeur

Association Livraisons d'histoire de l'architecture - LHA

Édition imprimée

Date de publication : 19 décembre 2012

ISSN : 1627-4970

\section{Référence électronique}

Anne Lefebvre et Olivier Liardet, « Louis Quételart et le phare de La Canche au Touquet », Livraisons de I'histoire de l'architecture [En ligne], 24 | 2012, mis en ligne le 16 avril 2015, consulté le 01 mai 2019. URL : http://journals.openedition.org//ha/103 ; DOI : 10.4000//ha.103

Ce document a été généré automatiquement le 1 mai 2019.

Tous droits réservés à l'Association LHA 


\title{
Louis Quételart et le phare de La Canche au Touquet
}

\author{
Louis Quételart's «Phare de la Canche » at Le Touquet \\ Louis Quételart und der Leuchtturm an der Mündung der Canche in Le Touquet
}

Anne Lefebvre et Olivier Liardet

1 Les côtes du littoral septentrional de la France ont de tout temps été réputées dangereuses: le relief, les embouchures de fleuves, les vents et les courants furent la cause de nombreux naufrages. Parmi les endroits les plus dangereux du littoral du Pas-deCalais, l'estuaire de la Canche présentait un cas particulièrement difficile, situé entre Boulogne-sur-Mer et l'embouchure de l'Authie faisant office de limite avec le département de la Somme.

2 A ces difficultés de navigation, deux phares vinrent remédier dans le grand mouvement d'éclairage des côtes de France initié en $1825^{1}$, précédant la naissance d'une des plus prestigieuses stations balnéaires du littoral français dont Louis Quételart fut l'un des promoteurs, participant à la conception du « style touquettois ». C'est tout naturellement à cet architecte célèbre sur la côte d'Opale que le service des Phares et balises, conscient des enjeux urbains liés à la reconstruction de cet équipement public, fit appel pour concevoir le phare touquettois par excellence.

\section{Le site, un lieu inhospitalier, et la construction des deux phares}

3 Au début de l'ère chrétienne, l'estuaire de la Canche était plus en amont que l'estuaire actuel $^{2}$. L'apport des sables dû aux vents d'ouest provoqua un relèvement de la grève et la mer se retira progressivement. Le nom du Touquet, c'est-à-dire le tournant, l'angle, n'apparût pas avant le moyen âge et s'appliqua alors à la partie extrême des garennes de Trépied sur la commune de Cucq. Jusqu'au XVe siècle, la pointe du Touquet n'avançait pas beaucoup plus loin que Trépied, mais à partir de cette date le littoral gagna progressivement sur la mer plusieurs kilomètres à cause de l'ensablement. Au XVII 
siècle, les hostilités entre la France et l'Espagne puis la peste décimèrent le village et le port de Trépied au profit de celui d'Etaples qui connaissait alors un fort développement. Jusqu'à la Révolution française, tout le domaine, constitué uniquement de dunes sauvages et arides, appartenait à l'abbaye Saint-Josse. Nationalisé à la Révolution, il fut conservé dans le domaine national.

$\mathrm{Au}$ début du XIXe siècle, seuls deux simples fanaux côté pointe du Touquet et un petit phare de l'autre côté de l'estuaire (démoli dans les années 1920), complété par un mât de sémaphore, signalaient l'entrée de la baie ${ }^{3}$. Depuis toujours, les naufrages étaient nombreux à cet endroit à cause de la faible profondeur à proximité du rivage. En janvier 1843, trois navires coulèrent en l'espace de trois jours, causant la mort de très nombreux marins. Cette triple catastrophe, largement commentée dans la presse, émut la population et attira enfin l'attention des pouvoirs publics, en provoquant un échange de points de vue entre les amirautés anglaise (nombre de bâtiments coulés étaient anglais) et française. À la suite de ces événements, la construction de deux phares fut décidée et confiée à l'ingénieur des Ponts et Chaussées Pigault de Beaupré secondé par le conducteur de travaux Denéchaux ${ }^{4}$. Cependant, plus de six années s'écoulèrent entre le moment de la construction et celui de la mise en service, et quatre naufrages furent encore à déplorer. Les deux phares furent enfin inaugurés le $1^{\text {er }}$ janvier 1852 (ill. 1).

\section{1 : Pigault de Beaupré, projet de construction des phares de la Canche}

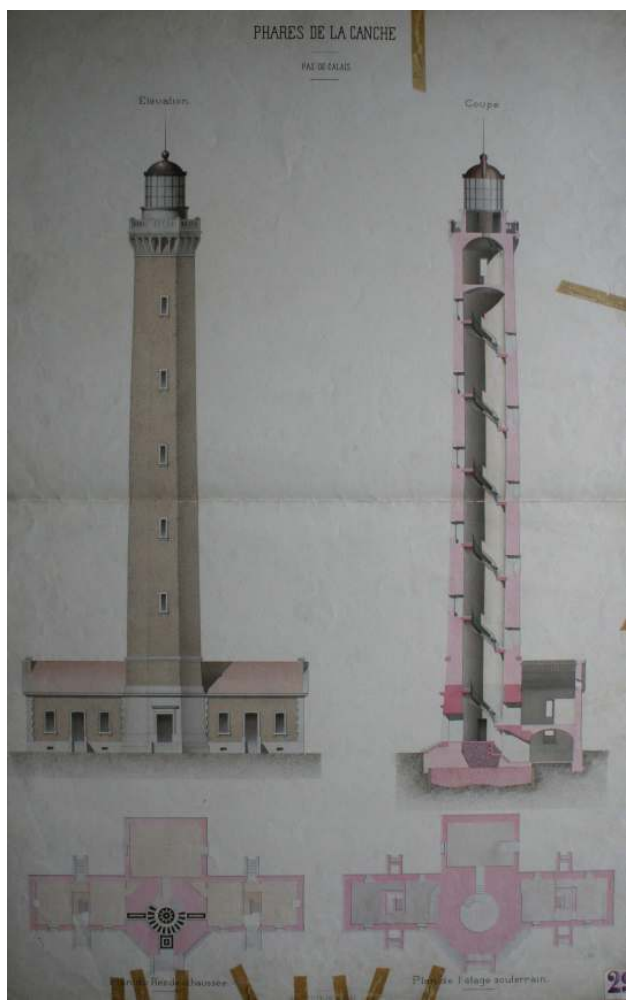

Élévation, coupe et plans, vers 1848, lavis sur papier, Arch. nat., CP/F 1417523

Cl. Olivier Liardet

Ces phares étaient édifiés bien en retrait du rivage, placés à 250 mètres l'un de l'autre sur un terrain rectangulaire de 600 mètres de long sur 300 mètres de large. Ils étaient à feu fixe avec une portée de 20 milles en mer $(37 \mathrm{~km})$. Au pied de chaque phare constitué d'un fût octogonal peint en blanc et d'un couronnement avec lanterne peint noir, un double 
corps de logis abritait le gardien et sa famille, tandis qu'un logement central était réservé au gardien chef en charge des deux phares. Une quinzaine de personnes au total vivait dans cet enclos.

\section{Naissance d'une station balnéaire (1819-1874)}

$6 \quad$ Au fil du temps, des particuliers s'étaient approprié des terrains domaniaux ${ }^{5}$. En 1819, les Ponts et chaussées dressèrent le plan des lieux afin d'évaluer les portions distraites. La concession de celles-ci fut décidée en 1822, puis rendue effective en 1827 (pour une part de 60 hectares sur un ensemble de 1.500 hectares depuis le retrait de la mer). Tout le domaine restant fut vendu en 1836 à un belge, M. Doms, qui, l'année suivante, ne l'avait toujours pas payé.

7 Il fut donc remis en vente et acquis en 1837 par Alphonse Daloz, notaire à Paris, et Alyon, qui s'employèrent à faire de l'élevage sur ces terres sans grand succès. En 1839, Alyon, tout en gardant une portion de ces terrains, en céda à Daloz une grande partie, et le reste à Marion (qui vendit à Daloz en 1850) et à de Naurois (qui vendit à Rigaud, beau-frère de Daloz, en 1855). Alyon vendit le restant de ses terres à Daloz en 1847. Daloz et Rigaud avaient ainsi acquis la maîtrise foncière et après une visite dans les Landes pour voir les résultats des plantations de pins par l'État, ils décidèrent de transformer le domaine en forêt. Ils plantèrent tout de suite des oyats et des pins maritimes pour fixer les dunes élevées, puis végétalisèrent les plaines. En 1858, le domaine fut définitivement partagé : Daloz garda la partie nord de 1.250 hectares, Rigaud le sud avec 350 hectares. Après quelques années, Daloz put planter des essences de feuillus plus fragiles comme des peupliers, des blancs de Hollande et des aulnes.

8 L'idée de créer une station balnéaire revint à Hippolyte de Villemessant, rédacteur en chef du journal Le Figaro, invité par Daloz à une partie de chasse en $1874^{6}$. Son projet de lotir une partie du domaine pour en faire un « Paris-Plage » ne put aboutir pour cause de maladie et de son décès quelques années plus tard, mais Daloz reprit l'idée à son compte : il fit niveler une partie du terrain par le géomètre Raymond Lens, puis découper le parcellaire en quadrillage d'îlots de douze parcelles chacun. Les deux premières villas furent construites en 1882, constituant le noyau d'un nouveau hameau de Cucq. Le rachat des terrains invendus en 1902 par la Société anglaise Le Touquet Syndicate Limited relança le développement de la station en lui donnant une grande extension vers la pointe qui n'avait cessé de s'avancer sur la mer, à tel point qu'en 1912 elle put être érigée en commune autonome sous le nom de "Le Touquet-Paris-Plage" avec 1.300 habitants permanents ${ }^{7}$. En 1904, dans le nouveau quartier des Oiseaux, extension de la station vers la pointe et la mer, on perça l'avenue des Phares, participant ainsi au nouvel urbanisme de la ville.

9 La Grande Guerre y amena de nombreux réfugiés belges et français, ainsi que la municipalité d'Ypres. Avant la Seconde Guerre Mondiale, la cité comptait 4.000 habitants l'hiver et 30.000 en haute saison et jouissait d'une grande réputation en rivalité avec Deauville ${ }^{8}$. L'Occupation fut intense pendant quatre années et c'est au cours de leur retraite dans la nuit du 2 au 3 septembre 1944 que les Allemands détruisirent les deux phares, 36 heures seulement avant l'arrivée de l'armée canadienne9. La tour sud fut sectionnée à 15 mètres du sol, tandis que la tour nord fut rasée entièrement. 


\section{La construction du phare actuel}

10 Un mois plus tard, en octobre 1944, on décida d'installer un feu provisoire sur le toit de l'hôtel de ville qui fut allumé le 15 octobre $1945^{10}$. Dès octobre 1944, le directeur du service des Phares et balises, A. de Rouville, après un entretien avec l'ingénieur Danel et le maire du Touquet, Jules Pouget, proposait de passer une convention entre les deux parties dans le but de reconstruire le phare : la commune apporterait une contribution financière aux travaux contre la cession d'une partie du terrain puisque l'on envisageait la reconstruction d'un seul phare au lieu des deux anciens détruits, libérant ainsi de l'espace vierge ${ }^{11}$. On prévoyait de conserver néanmoins une surface suffisante de terrain pour les cultures vivrières des gardiens à cause du coût de la vie dans la station balnéaire, et de déplacer le bâtiment central (maison du gardien chef) qui était pourtant restaurable.

11 Le directeur suggérait également de prendre « le concours d'un architecte local capable de donner aux nouvelles constructions un aspect en harmonie avec le cadre de la station de Paris-Plage $~{ }^{12}$. L'ingénieur subdivisionnaire Danel proposait de se " mettre en rapport avec M. Louis Quételart, architecte, dès son retour au Touquet Paris-Plage $»^{13}$, ce qu'il fit le jour même, choix approuvé par le directeur le 17 novembre $1944^{14}$.

\section{Louis Quételart (1888-1950), architecte de bord de mer}

L'architecte Louis Léon Joseph Quételart naquit à Berck-sur-Mer le 27 mars $1888^{15}$ (ill. 2). 


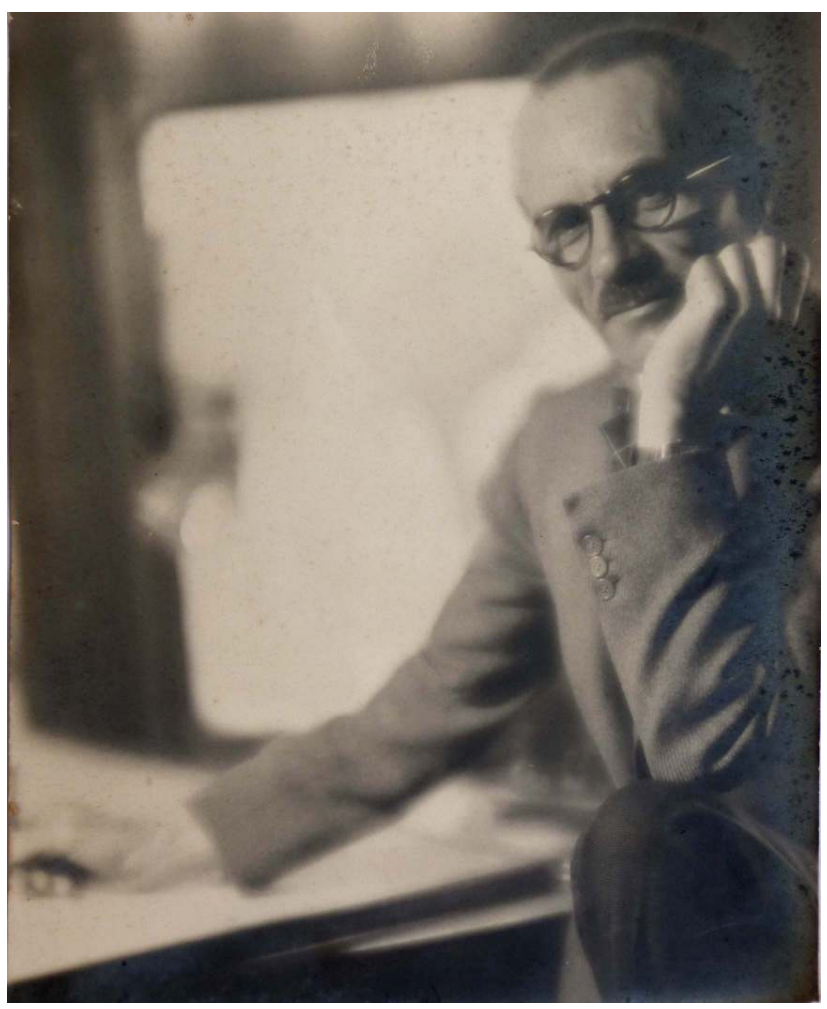

S.d., tirage photographique, Agence Quételart (Le Touquet)

Cl. Olivier Liardet.

Issu d'un milieu modeste, il ne put poursuivre sa scolarité après le certificat d'études primaires. Autodidacte, il entra à l'âge de 14 ans dans le cabinet de l'architecte berckois Gaston Bellêtre, spécialisé dans les habitations balnéaires, puis dans l'entreprise de travaux publics Charbonnier, également de Berck. En 1907, fort de son expérience, il intégra l'agence d'Albert Pouthier, architecte au Touquet, tournée vers dans la résidence secondaire de luxe, puis en 1912 créa sa propre agence dans la même ville. Son œuvre débute par une trilogie de villas de taille modeste construites pour sa famille: Fadette, Mon Chaume et Nine.

Il prit un rôle actif dans la reconstruction de la région, mais ne disposant pas d'un diplôme d'architecte, il dut s'associer pour participer à la commande publique. Sa connaissance des milieux locaux en fit un collaborateur précieux, il avait entre autres de bonnes relations avec l'architecte Louis-Marie Cordonnier. En 1920, il ouvrit une agence à Béthune avec l'architecte parisien Pierre Ragois, que vint rejoindre l'année suivante André Pavlovsky. Ensemble, ils travaillèrent à la reconstruction de Vieille-Chapelle et d'Annezin dans le Pas-de-Calais, de Méteren et La Gorgue dans le Nord, travaillant alors plutôt dans le style de la Renaissance flamande matinée d'Art déco.

Son enracinement dans la pratique locale lui permit de contribuer à la création du « style touquettois moderne", ferment de l'identité urbaine du Touquet. Caractérisé par des façades à pignons ou doubles pignons souvent percés d'oculi, des toitures pentues, des retombées d'arcs sans piédroits, son style est facilement identifiable dans la trentaine de villas qu'il a réalisées. Dès le début des années 1920, il était capable de concevoir des plans originaux tout en gardant une apparence séduisante pour ses riches clients, ce que 
beaucoup soulignaient: "[Quételart] a marqué de son talent très personnel, un pourcentage imposant des plus jolies constructions de la forêt. Et c'est bien grâce à lui que telle personnalité artistique américaine pouvait nous dire un jour : «Les villas du Touquet sont les plus jolies maisons que j'ai jamais vues », et telle autre, le lendemain : "Les villas du Touquet sont tellement séduisantes qu'on voudrait pouvoir les emporter " ${ }^{16}$.

Son œuvre développe un style varié et personnel qui s'épanouit dès 1925 avec la villa Les Mutins ${ }^{17}$ et atteint sa maturité avec une villa construite à Rambouillet en 1933 (Clairbois). La majorité de sa production concerne l'habitat privé : villas Pomme d'Api en $1923^{18}$, Bic en Coin en $1925^{19}$ (ill. 3), Chat perché et Scarabée ${ }^{20}$ toutes deux en 1927, La Prairie 1928, mais aussi Melki, L'Outarde, Ton Taine, Fiordaliso, Mon Pignon, Sauvageonne, Poppy, Datchia, L'Énigme, Venvole, etc. Au Touquet, il réalisa aussi des équipements : les premier et second hôtels Scampolo (1924, 1926-30), l'aérogare, le plongeoir de la piscine ou encore le Casino.

\section{3 : Le Touquet, Villa Bic en Coin, vue actuelle}

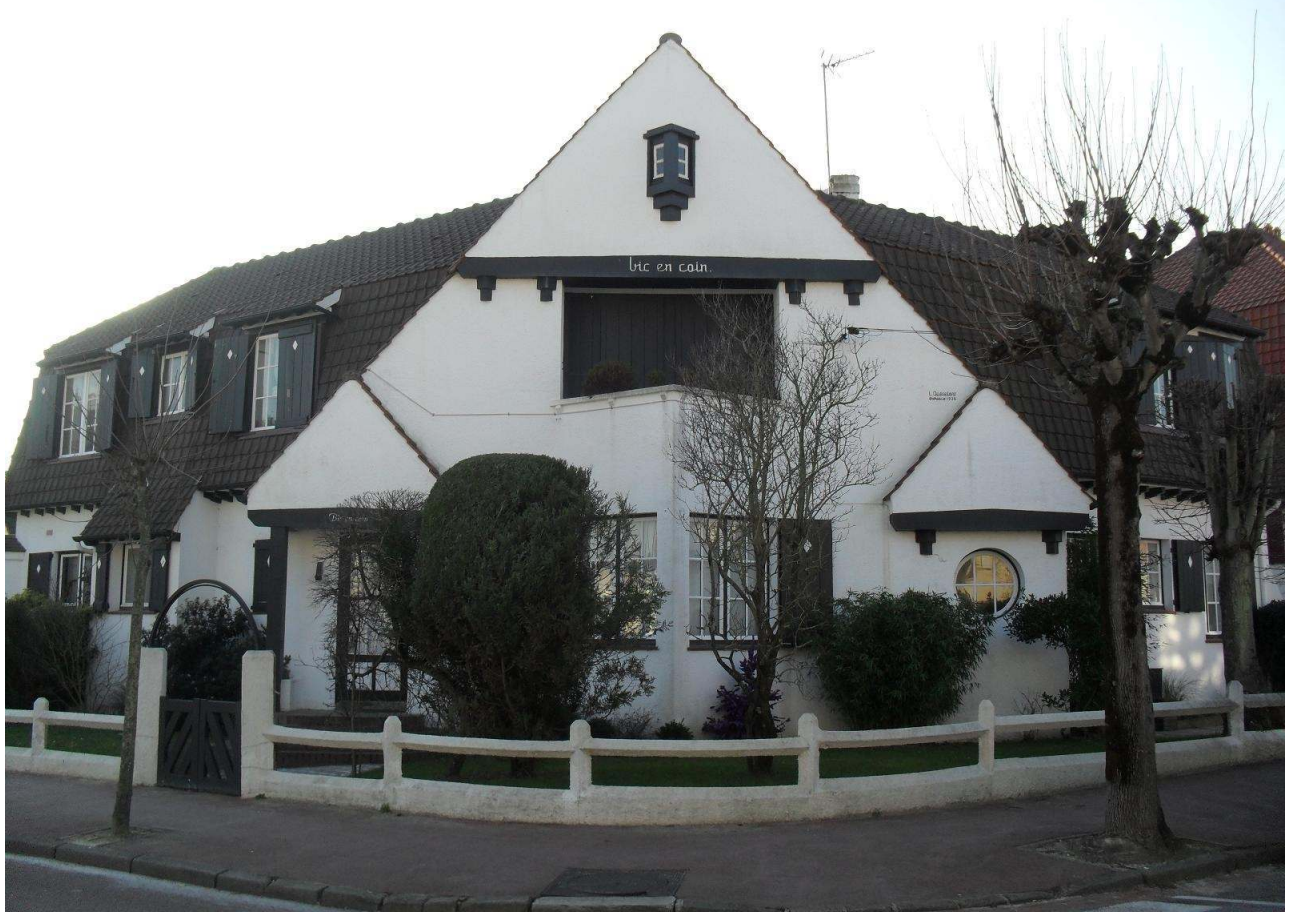

Cl. J.-B. Cousin

Il participa à l'Exposition universelle de Paris en 1937 (pavillon de la Flandre, du Hainaut et de l'Artois avec Jacques et André Barbotin et J.-L. Morel, au sein du Centre régional en bordure du Champs de Mars), puis à l'Exposition de Roubaix en 1939. Membre de nombreuses associations professionnelles tel le Conseil de l'Ordre des Architectes, il en présidait plusieurs comme le Comité des Architectes Agréés du Pas-de-Calais et le Syndicat des Architectes du Pas-de-Calais. Adjoint à l'Architecte en Chef du Département, il fut également adjoint au Maire du Touquet.

Son œuvre construite et l'intérêt de ses écrits (diffusion de ses projets dans les revues et publications d'architecture) font de lui une des figures marquantes de l'architecture 
régionaliste de l'entre-deux-guerres ${ }^{21}$. Après la Seconde Guerre Mondiale, la construction du phare apparaissait pour lui comme le "bâton de maréchal» de sa carrière d'architecte. Pour l'occasion, il s'adjoignit les services de son fils Pierre Quételart, alors élève de $2^{\text {e }}$ classe à l'École nationale des Beaux-Arts à Paris, d'Adolphe Cailleau, chef d'agence de son cabinet, et de Gérard Peyrot, dessinateur-projeteur. Il décéda subitement au Touquet le 19 août 1950. Son décès fut un événement marquant de l'après-guerre touquettois comme le soulignait un journaliste: «Extrêmement sympathique, le défunt était en même temps qu'un grand artiste à qui Le Touquet doit son style tout particulier, une éminence de l'art architectural et aussi un écrivain de talent. Il laisse une œuvre utile qui durera et qui est la plus belle qui puisse honorer la mémoire d'un homme. L'une de ses plus belles réalisations fut le Casino du Touquet. On lui doit aussi de magnifiques villas en forêt et parmi les derniers projets qu'il réalisa, le phare, véritable merveille en son genre $»^{22}$.

\section{Le programme du nouveau phare}

Danel, via le Service central, transmit les éléments d'un programme à l'architecte le 25 novembre $1944^{23}$ : la base de la tour sud (derrière l'hôtel Westminster), de teinte blanche, pouvait être réutilisée pour le nouveau phare; le fût serait recouvert d'un parement en briques rouges de préférence à un enduit; deux logements pour les familles des gardiens de quatre pièces chacun avec des communs étaient prévus ainsi que trois pièces pour les ingénieurs de passage. Pour ce faire, le bâtiment central construit en 1852 pour le logement du gardien en chef ayant subsisté, il convenait de le réutiliser au moins provisoirement (logement de gardien et pièces réservées aux ingénieurs de passage). Quant au second pavillon à construire, il devait être proche du phare, mais détaché pour bien marquer l'élancement de ce dernier.

Le dossier préparatoire de la reconstruction fut transmis par Quételart le 9 février 1946 à Danel avec deux variantes, toutes les deux situées en centre-ville sur l'ancien terrain des phares $^{24}$ : la première (A) prévoyait la reconstruction sur la base de l'ancienne tour sud, la seconde (B) une reconstruction au centre du terrain (ill. 4) 
III. 4 : L. Quételart, plan de situation avec les deux variantes du projet d'implantation du nouveau phare

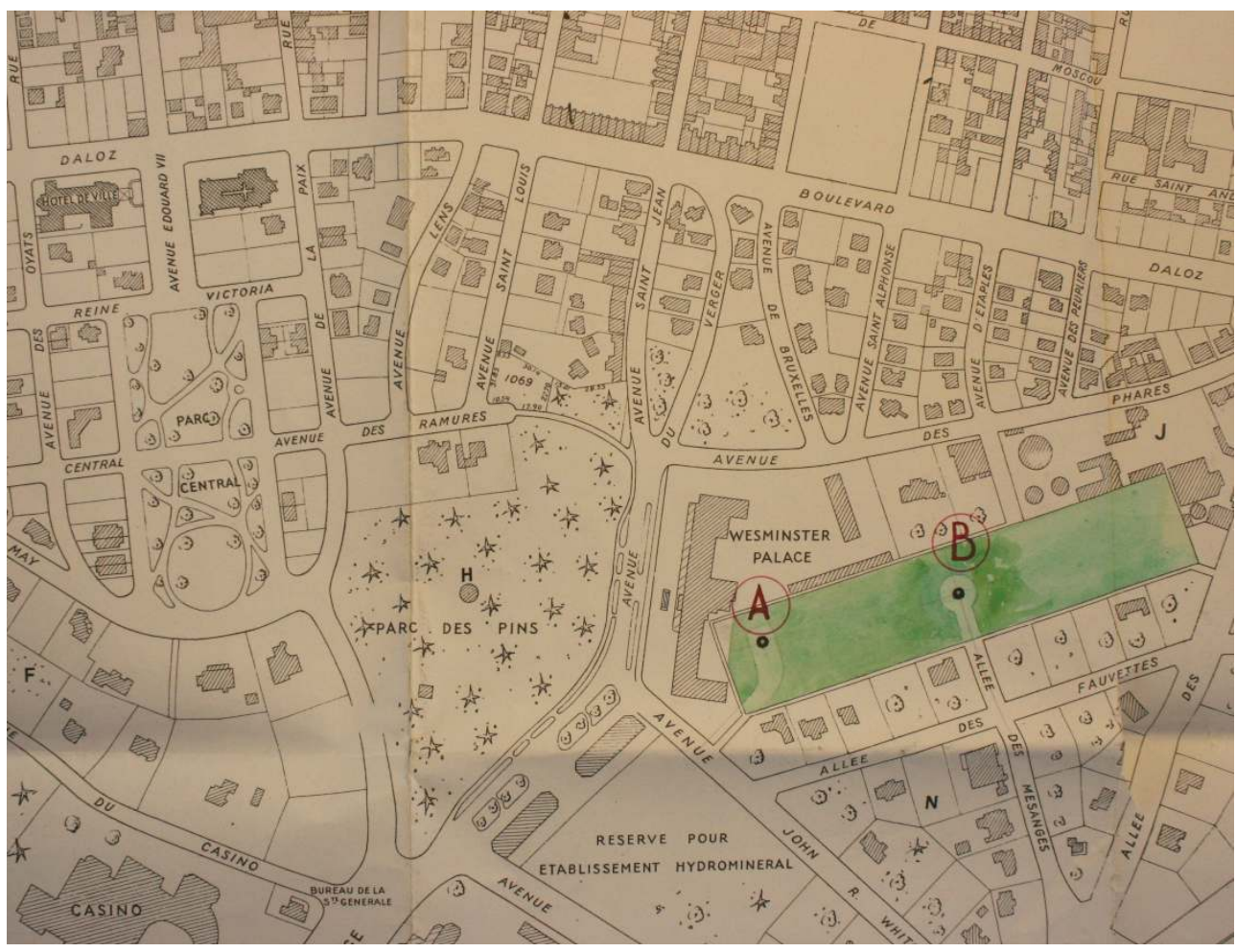

Tirage coloré, Arch. nat., F ${ }^{14} 19875$

Cl. Olivier Liardet

21 Une troisième implantation (C) avait cependant été envisagée à la pointe du Touquet, mais Quételart, sans la repousser, la trouvait problématique pour la construction des logements et jardins, mieux abrités dans la ville. Quant au phare, Quételart donnait sa préférence à une forme terminale « où le chapiteau s'évase en prolongement du fût, avec piliers et arcs entre les galeries, et lanterne vitrée au maximum $»^{25}$.

\section{L'avant-projet de Quételart et la nouvelle situation du phare}

La base ayant été trop ébranlée par les explosions et les pierres mal taillées au moment de la mise en œuvre, on prit l'option d'une reconstruction totale de cette base au vu de la faible économie réalisée, environ $10 \%$ du total au plus :

«Convient-il dans le seul dessein de réaliser une économie d'importance limitée de décider la reconstruction d'un édifice sur son ancien emplacement en sacrifiant définitivement l'esthétique générale de l'établissement? L'Architecte ne le pense pas et nous ne le pensons pas davantage. Aussi sommes-nous d'avis d'abandonner définitivement cette solution qui alimenterait indéfiniment une source de critiques d'autant plus fâcheuses pour le bon renom de l'Administration Française que la station du Touquet-Paris-Plage est fréquentée par une clientèle internationale de caractère généralement relevé (Aristocratie et hommes d'affaires anglais, diplomates, industriels du nord de la France, etc.) $»^{26}$. 
Le seul inconvénient de cette solution était la réduction du terrain à rétrocéder à la ville contre le financement partiel de la construction. De ce fait, l'emplacement au centre du terrain, comme le proposait Quételart, devenait une évidence: "Enfin puisqu'il faut reconstruire le soubassement, on a pensé que le déplacement du phare peut être envisagé, sauf à considérer le prix d'une nouvelle fondation. Le plan de situation prévoit le nouveau phare sur l'axe longitudinal du terrain et en prolongement de l'axe de l'allée des Mésanges, de manière qu'il soit vu du carrefour formé par la Route Nationale 318, l'allée des Rossignols, l'allée des Mésanges et l'avenue John Whitley - solution satisfaisante du point de vue urbanistique $»^{27}$. Le service des Ponts et chaussées fut autorisé à aliéner deux parcelles de 25 ares chacune aux extrémités de l'ensemble réduisant l'emprise à 1 hectare 14 ares et 74 centiares $^{28}$.

\section{Le projet définitif}

Le projet fut examiné très rapidement par les ingénieurs locaux et l'ingénieur subdivisionnaire Danel donna ses conclusions ${ }^{29}$ : en accord avec l'architecte sur le choix de l'emplacement et les aspects généraux de la tour, il préférait comme Quételart la solution d'une forme évasée au lieu de deux plates-formes superposées très séparées (ill. 5).

III. 5 : L. Quételart, projet de nouveau phare, détail de la partie supérieure avec fût évasé

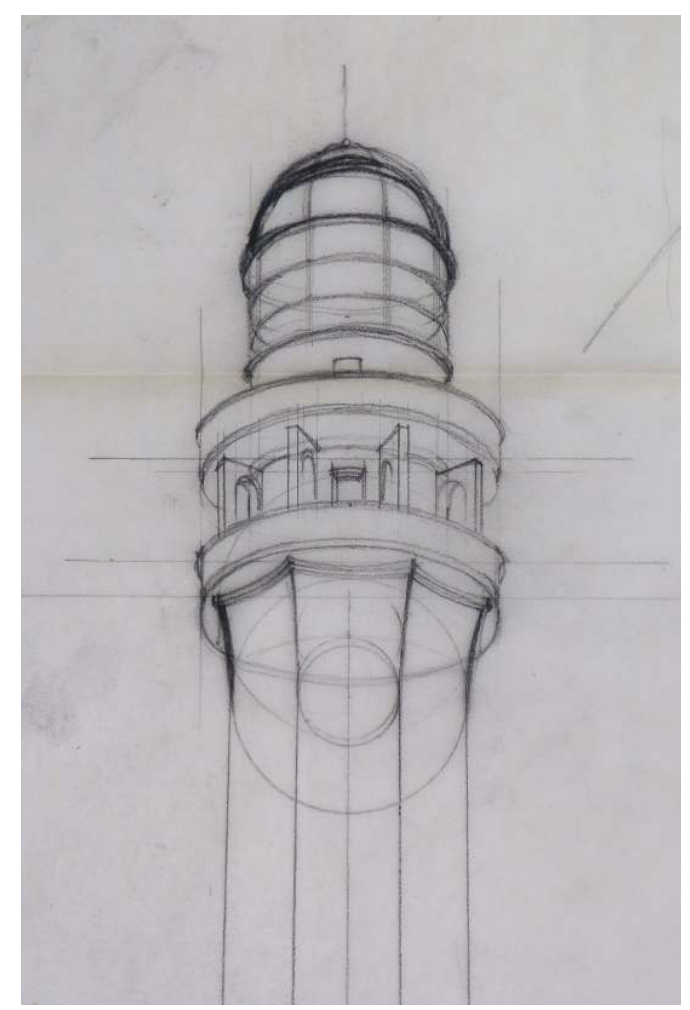

S.d., crayon sur calque, Agence Quételart (Le Touquet)

Cl. Olivier Liardet 
L'ingénieur d'arrondissement Leclercq adopta ces conclusions le 6 mars, mais revenait à la solution des deux plates-formes et à un éclairage plus traditionnel de la lanterne avec les parois seules verticales vitrées (ill. 6).

III. 6 : L. Quételart, projet de nouveau phare, détail de la partie supérieure adoptée

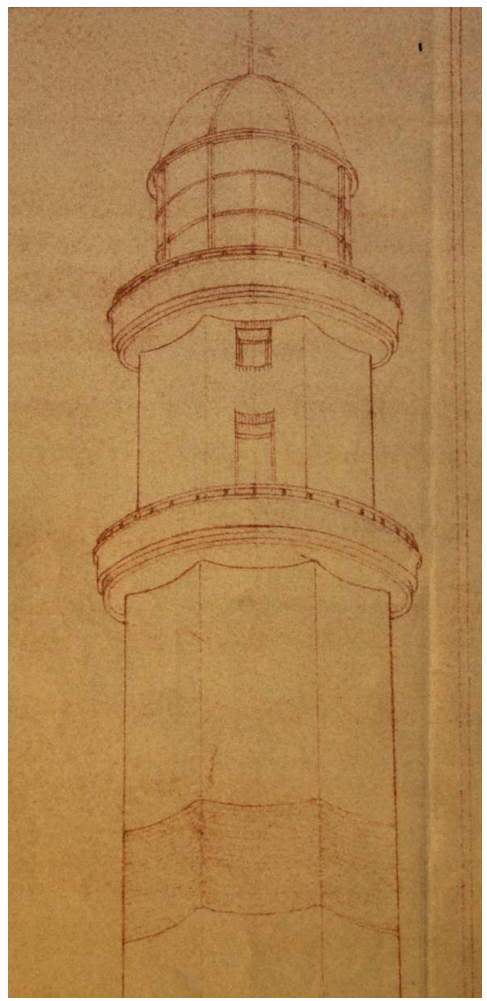

14 février 1946, tirage, Arch. nat., F ${ }^{14} 19875$

Cl. Olivier Liardet

L'ingénieur en chef du département de Viry suivit les conclusions de Leclercq le 11 mars suivant, en précisant qu'il préférait les garde-corps pleins au lieu des rambardes ajourées. Le service parisien autorisa la mise au point du projet définitif le 15 mai 1946 en adoptant les conclusions générales du rapport des ingénieurs sur le choix de l'emplacement, s'en remettant à eux et à l'architecte pour le choix de la forme de la partie supérieure, mais en insistant cependant pour que le garde-corps de l'escalier soit réalisé dans un beau matériau, le fer forgé de préférence : «Une fonte moulée risque d'être assez disgracieuse et nous avons trop souvent consenti des rampes modestes qui déparent à elles seules une construction de belle venue $\aleph^{30}$. Parallèlement, Georges Tourry avait également été chargé du projet de reconstruction, mais ses projets ne semblent pas avoir été conservés. Il s'occupa ensuite de celle du phare de Berck en béton précontraint ${ }^{31}$.

Le projet définitif de reconstruction fut dressé le 15 octobre 1946 et remis le 18 novembre par Quételart ${ }^{32}$. Le phare adoptait la position centrale souhaitée par tous agrémenté d'un jardin octogonal reprenant la forme de la tour. Pour les fondations, suivant les suggestions des ingénieurs, Quételart y incluait des palplanches à l'intérieur desquelles les fondations seraient incluses afin de créer un coffrage étanche et limiter les infiltrations de la nappe phréatique. Pour le reste, il intégrait toutes les demandes des ingénieurs, garde-corps pleins pour les galeries, coupole traditionnelle, soubassement en pierre de taille porté à 4,50 mètres. Le fût de la tour devait mesurer 45 mètres de hauteur 
depuis le niveau du sol pour une section de 6,40 mètres de diamètre extérieur à la base et 5,20 mètres au sommet. Cette diminution progressive du diamètre favorisait l'impression d'élancement. Si le soubassement était octogonal avec des faces plates, le fût lui-même avait la particularité d'avoir des faces concaves comme une colonne cannelée renforçant l'élancement de la tour et lui conférant un aérodynamisme recherché.

\section{Devis et coût}

Le devis estimatif fut dressé le $1^{\text {er }}$ février 1947 par Quételart pour un montant total de 28.787.830 francs, soit près de 17 millions uniquement pour le phare. Le service des Ponts et chaussées proposait de le réduire à 27 millions mais ajoutait 2,5 millions pour les imprévus non inclus par l'architecte.

Les ingénieurs du Pas-de-Calais examinèrent le projet les 17 et 19 février suivants et le transmirent à Paris pour décision ${ }^{33}$. Le 4 mars, de Rouville, directeur du service, autorisa le projet avec les modifications proposées par l'architecte et les ingénieurs, en particulier la réalisation de la superstructure de la tour avec ses plates-formes devant être exécutées entièrement en pierre y compris le parapet, ce qui ne sera pas le cas finalement ${ }^{34}$. Le 13 mars le ministre des Travaux publics et des transports donna son accord définitif à la reconstruction du phare, en excluant toutefois la construction d'un des logements de gardien, du logement des ingénieurs et du monte-charge, ce qui réduisait le montant du crédit à 26 millions ${ }^{35}$.

La hausse considérable des prix des matériaux obligea le ministre à donner une rallonge au crédit prévu pour le porter à 42 millions ${ }^{36}$, puis une seconde, en février 1949, portant le montant à 51.300 .000 francs $^{37}$, puis à 52.650 .000 francs $^{38}$. Au final, la reconstruction du phare coûta près du double du montant initial des travaux. Les travaux furent adjugés à l'entreprise Le Génie civil de Lens les 26 et 30 septembre 1947 (Rouger, présidentdirecteur, et Caridroit, ingénieur représentant pour la région) et commencèrent peu après sous la direction des ingénieurs du Service maritime avec le conseil de Quételart (ill. 7). Ils ne furent achevés qu'en 1952 alors que le feu était allumé depuis septembre 1951. 


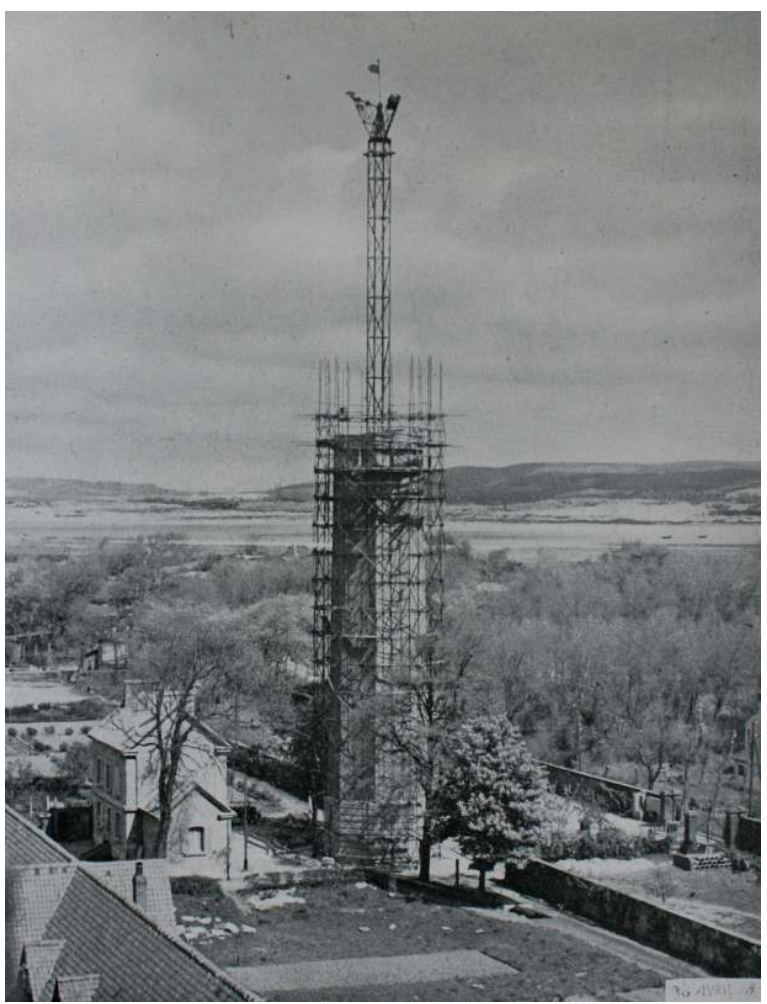

30 avril 1949, publiée dans L. Quéterlart, « La reconstruction du phare du Touquet », La Construction moderne, sept. 1949, p. 326

Cl. Olivier Liardet

\section{Choix des matériaux et techniques constructives}

Les fondations devaient être posées sur un radier en béton armé, le sous-sol recevant une fosse de $1,50 \mathrm{~m}$ par $1,50 \mathrm{~m}$ et $1,20 \mathrm{~m}$ de hauteur ${ }^{39}$. Le soubassement, à 3,50 mètres audessus du sol, réutilisait les pierres de Marquise provenant de la démolition du soubassement de la tour sud, doublées par des parements en pierre neuve de même provenance taillées en bossage, et le socle était traité en bacs à fleurs débordant, les marches d'accès étaient dans la même pierre ${ }^{40}$ (ill. 8). 
III. 8 : L. Quételart, détail de l'élévation du soubassement avec la porte d'entrée du phare

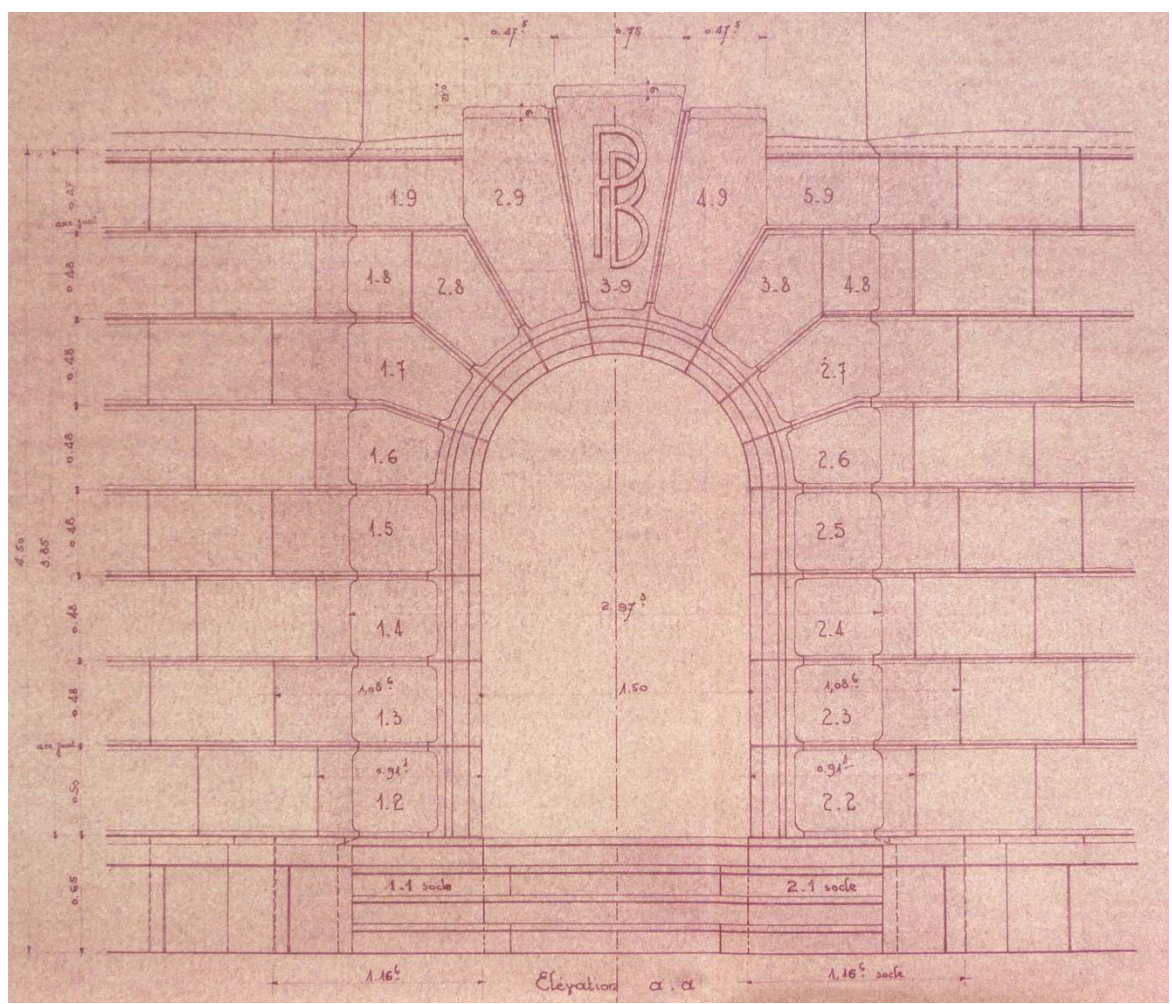

S.d., tirage, Agence Quételart (Le Touquet)

Cl. Olivier Liardet

Pour le vestibule, Quételart prévoyait un dallage en marbre d'Hydrequent avec une étoile à cinq branches, symbole des phares et balises ${ }^{41}$. Le fût de la tour fut construit en briques rouges ordinaires provenant d'Attin près du Touquet, avec pour le parement extérieur un type de briques particulier. Le choix de ces briques de parements ne cessa de préoccuper les ingénieurs et l'architecte, cherchant dans la région (Haubourdin, Écuelles) ou en Belgique (Ostende) des échantillons afin de trouver le meilleur rapport qualité-prix. En avril 1948, les ingénieurs firent réaliser des essais de tenue de "briques Silésia " provenant d'un fabricant de Fouquereuil dans le Pas-de-Calais (Briques et Céramiques de Fouquereuil, Paul Vandecasteele) ${ }^{42}$. Ces essais furent concluants pour la compression, la porosité et la gélivité. L'entreprise pouvait également fabriquer des briques d'angle pour éviter les joints sur les arêtes du fût (ill. 9). 


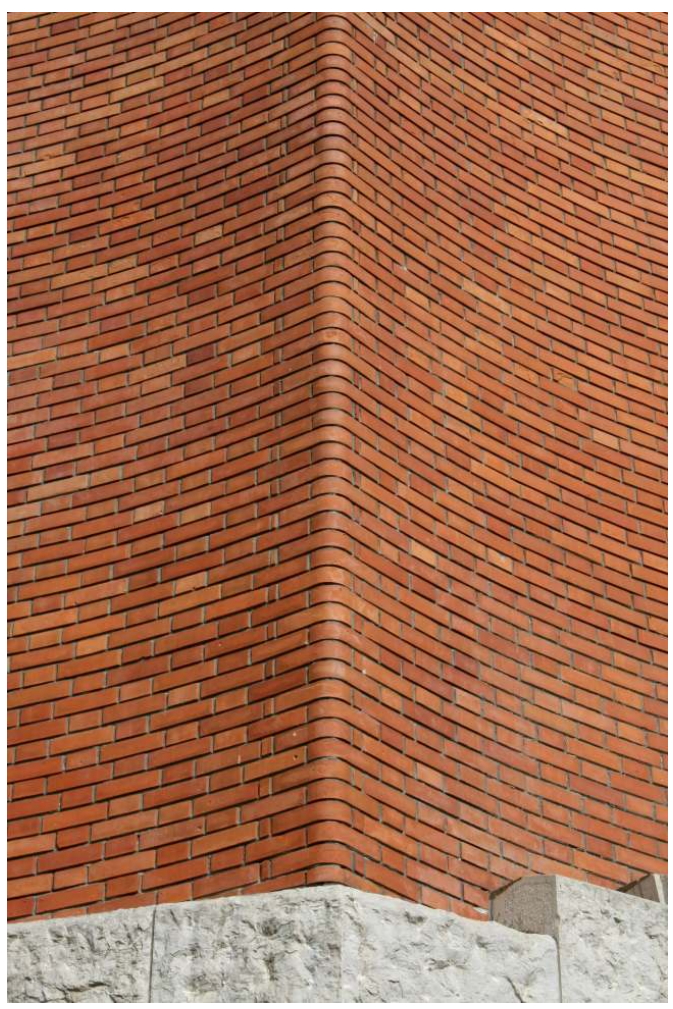

Cl. Olivier Liardet

Le service central autorisa ce choix le 28 juin 1948 avec des briques jaune orange pour les deux faces, intérieure et extérieure, tout en s'interrogeant en marge : « est-ce beau ? ${ }^{43}$. Entre les cotes 34,80 et 38,80 , un bandeau de 4 mètres fut réalisé en briques d'un ton brun Van Dyck pour la signalisation de jour.

34 Sept frettes en béton armé raidirent la construction sur toute la hauteur du fût. Les briques $\mathrm{du}$ "chapiteau » du fût butaient sous la ceinture en béton de la première plateforme accueillant la chambre de veille, tandis que la seconde supportait la machinerie de la lanterne (cuve à mercure) ${ }^{44}$. Ces plateformes en béton armé furent recouvertes de différents matériaux pour les protéger des intempéries : les sols des deux galeries en pierre du Boulonnais formant cuvette sur une feuille d'étanchéité en plomb et les parapets dans la même pierre avec bandeau et larmier saillant externes. Pour solidariser entre elles ces deux plateformes, on plaça huit poteaux en béton armé, les parements extérieur et intérieur en pierre du Boulonnais en constituant les murs ${ }^{45}$.

L'intérieur desservi par un très imposant escalier en spirale de 274 marches montait jusqu'à la chambre de service en béton armé préfabriqué bouchardé sur les parties visibles. Le dessus des marches était recouvert de plaques de marbre du Boulonnais d'une épaisseur de $4 \mathrm{~cm}$, une rambarde en fer et cuivre protégeant du vide central. La volée supérieure entre les deux plateformes était réalisée en béton armé monolithe et les marches (275 à 297) étaient bouchardées et revêtues de marbre. Depuis la chambre de la lanterne, un escalier métallique permettait l'accès à la lanterne en bronze avec son dispositif supportant le système rotatif de l'optique, présentant les caractéristiques générales traditionnelles des lanternes depuis les années 1820 : circulaire, partie basse 
pleine avec des évents, partie haute vitrée, coupole hémisphérique pleine, étroite galerie extérieure (ill. 10).

III. 10 : L. Quételart, plans et coupe de la partie supérieure du phare avec les plate-formes et la lanterne

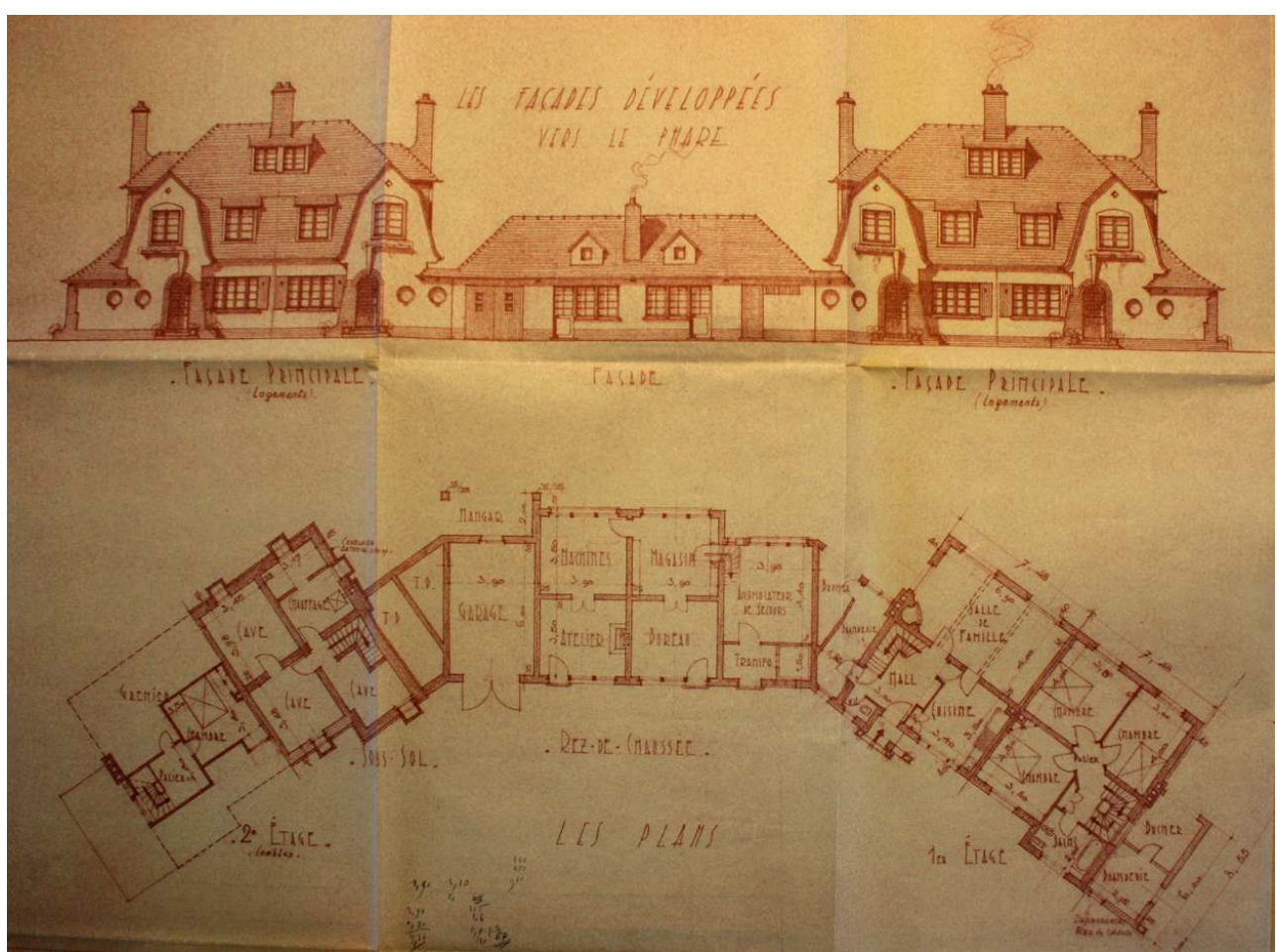

15 octobre 1945, tirage, Arch. nat., F ${ }^{14} 19875$

Cl. Olivier Liardet

La main-courante des parapets des galeries fut également exécutée en bronze. Les portes et menuiseries furent réalisées en chêne avec des ferrures de bronze. Le prix des fondations et du soubassement en pierre montait à eux seuls à 3.077.893,65 francs. La qualité des matériaux choisis après plusieurs années de réflexions et d'essais, outre le côté esthétique mis en avant dès la construction, a assuré la pérennité du bâtiment.

\section{Les annexes}

37 Dès le départ, Quételart envisagea une reconstruction complète de l'ensemble formé par le phare et ses annexes. Multipliant les projets, il proposa à chaque étape d'élaboration des variations sur le thème de la villa touquettoise. Dans un premier temps, il imagina deux maisons doubles séparées et encadrant la tour sud conservée. Elles présentaient un plan ramassé n'excluant pas le pittoresque dans l'animation des masses : sur la façade principale les pignons de chaque habitation se trouvaient englobés dans un grand et large pignon, tandis qu'à l'arrière ce grand pignon couvrait un pignon plus petit, les deux s'emboitant comme des poupées russes ${ }^{46}$. Il réutilisa les mêmes pavillons doubles dans une seconde version de son projet global avec une implantation similaire autour du phare placé au centre du terrain ${ }^{47}$.

Une fois l'aspect général de la tour choisi, Quételart proposa une version des annexes prolongeant son style si particulier au-delà de la guerre. Le long bâtiment des 
dépendances et logements se trouvait au sud de la tour placée au centre du terrain suivant le plan en octogone du jardin sur ses trois pans $\operatorname{sud}^{48}$. L'architecte donnait ainsi un cadre au phare et créait une barrière pour rendre privatifs les jardins et potagers des familles de gardiens résidant dans deux pavillons de deux logements jumelés chacun et réunis par une aile plus basse pour la machinerie et le service. Dans cette ultime version, Quételart retrouvait les accents de ses propres projets touquettois d'avant-guerre: dans une volumétrie pittoresque, il développait tous les éléments de ses fameuses villas. Sur de longs toits pentus et débordants, il ouvrait de petits pignons à double pentes et surtout perçait de hautes cheminées recouvertes de tuiles plates, sans oublier de multiplier les lucarnes sur deux niveaux. Porches à jambage taluté et oculi complétaient cette ode au meilleur du régionalisme finissant (ill. 11).

III. 11 : L. Quételart, élévation et plan des annexes projetées

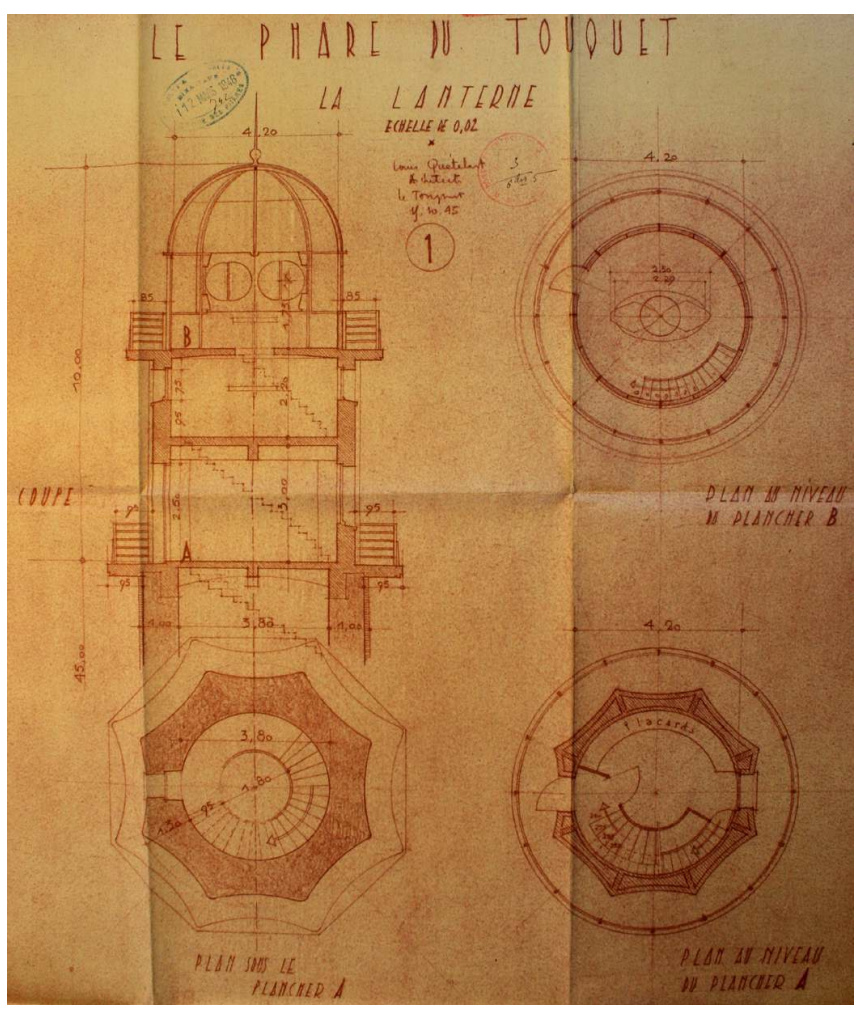

15 octobre 1946, tirage, Arch. nat., F ${ }^{14} 19875$

Cl. Olivier Liardet logement du gardien en chef, se trouvait initialement entre les deux anciens phares ${ }^{49}$. Petit pavillon de style néoclassique, il apparait très traditionnel dans l'architecture de gardiennage au XIXe siècle comme les maisons forestières des domaines de l'État en Sologne ou d'île-de-France (Versailles, Saint-Germain-en-Laye, Marly, etc.). Le pavillon central, d'une travée d'épaisseur et trois de largeur, de deux étages carrés sous un comble à deux pentes, était accompagné de deux courtes ailes d'un seul niveau. Il fut décidé, en octobre 1948 , de le conserver et le restaurer ${ }^{50}$. Il se trouvait désormais à l'arrière du nouveau phare. 
41 Il ne restait donc plus qu'à construire un seul logement et à l'harmoniser avec celui existant non démoli, puis de faire quelques travaux au bâtiment de service construit dans le courant de la deuxième moitié du XIX ${ }^{\mathrm{e}}$ siècle afin de l'utiliser encore pendant quelques années. Quételart en dressa les plans en février $1949^{51}$.

\section{Phare et régionalisme balnéaire : l'inscription dans le paysage touquettois}

«Ce nom, pour ceux qui sont fidèles à nos rivages, c'est l'évocation d'un style, le style même du Touquet. Ce très grand architecte et non moins grand artiste a su dégager de l'architecture, - cet art exact, - sa personnalité. Puis il en fit celle du Touquet $»^{52}$.

42 Ainsi Quételart apparaît-il de son vivant, comme après sa mort, tel l'architecte-créateur non seulement d'un style très personnel, mais surtout du style propre à cette station balnéaire, réalisant même les bancs-jardinières jalonnant le parcours urbain ${ }^{53}$. Pourtant sa dernière œuvre, le phare de la Canche, rompt avec sa tradition personnelle. Contrairement à l'architecture des villas balnéaires dont il s'était fait le spécialiste, mêlant pittoresque et influences régionales, rigueur de la mise en œuvre des matériaux et confort moderne, ce premier et unique chantier réalisé pour le compte de l'État ${ }^{54}$ montrait une mise en perspective dans la longue tradition des phares depuis la fin du XVIII ${ }^{\mathrm{e}}$ siècle.

43 Il alliait dans cet ouvrage l'approche réaliste des matériaux traditionnels et un usage modéré du béton armé "protégé » par une peau de pierre locale. Mais il faisait aussi preuve d'une analyse savante de l'histoire de l'architecture. Colonne plus que tour, le phare de la Canche se rappelait ainsi de la théorie des ordres de l'architecture classique. Le phare devenait un fût cannelé de colonne à l'antique dont la longue trajectoire à travers le ciel le faisait passer de la tradition, avec sa base de pierres pittoresquement rustiquées, à la modernité d'un chapiteau tout entier dédié à la lumière et sa technologie (ill. 12). 


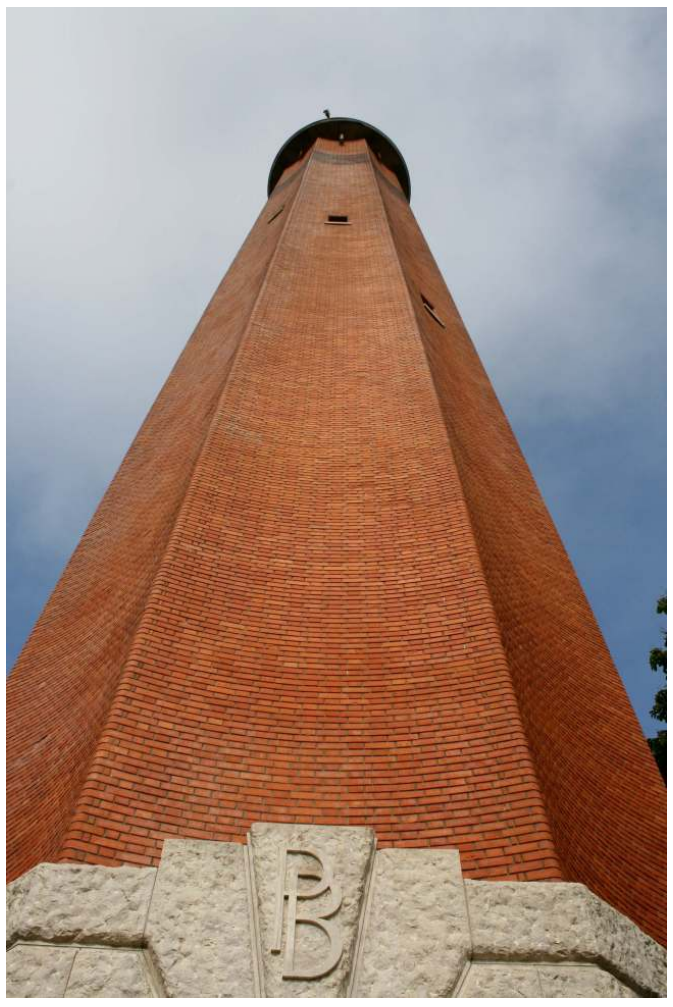

Cl. Olivier Liardet spécificités du climat, caractéristique de ses maisons ${ }^{55}$, Quételart s'était peut-être lancé pour la première fois dans un exercice conceptuel que l'enseignement académique n'aurait pas démenti. Il faisait ainsi l'épreuve à la fin de sa vie de ce qui lui avait manqué à ses débuts, et montrait avec maestria qu'il aurait sans doute pu remporter le premier prix.

Après la Libération, Quételart se consacra activement à la reconstruction de la ville malmenée par les Allemands, mais ce surmenage, auquel la reconstruction du phare participait, devait lui être fatal comme l'indiquaient plusieurs chroniqueurs au moment de sa mort : «Sa puissance de travail était, en effet, à la mesure de son imagination et de son talent qu'il avait cultivé tout seul; et si, depuis quelques années, depuis la fin de la guerre qui l'avait frappé dans affections les plus chères, il devait lutter jour après jour contre une maladie sournoise et déprimante, il n'en continuait pas moins à faire face à la tâche écrasante qui lui valait sa notoriété $»^{56}$. Prolongeant l'œuvre du maître, le cabinet Quételart réalisa la clôture des jardins en 1956 en concevant le portail d'entrée reproduisant les bossages rustiques du soubassement dans ses quatre piliers ${ }^{57}$.

L'histoire de la reconstruction du phare de la Canche au Touquet est révélatrice d'une entente parfaite entre une municipalité avertie, un commanditaire conscient des enjeux, l'architecte et les ingénieurs chargés de la réalisation, animés par la volonté de créer un monument en harmonie architecturale avec l'esprit de la station balnéaire. Alors qu'à l'origine, les deux phares jumeaux se trouvaient isolés dans les dunes, la mise en situation urbaine après la guerre de l'ensemble constitué du nouveau phare entouré d'un jardin 
avec la maison du gardien en chef et le bâtiment des machines, que l'on découvre au bout d'une longue allée, participe complètement à l'image de la ville (ill. 13).

III. 13 : Vue générale actuelle du phare au centre de son jardin avec la maison du gardien chef de 1852 et les dunes de l'estuaire dans le lointain

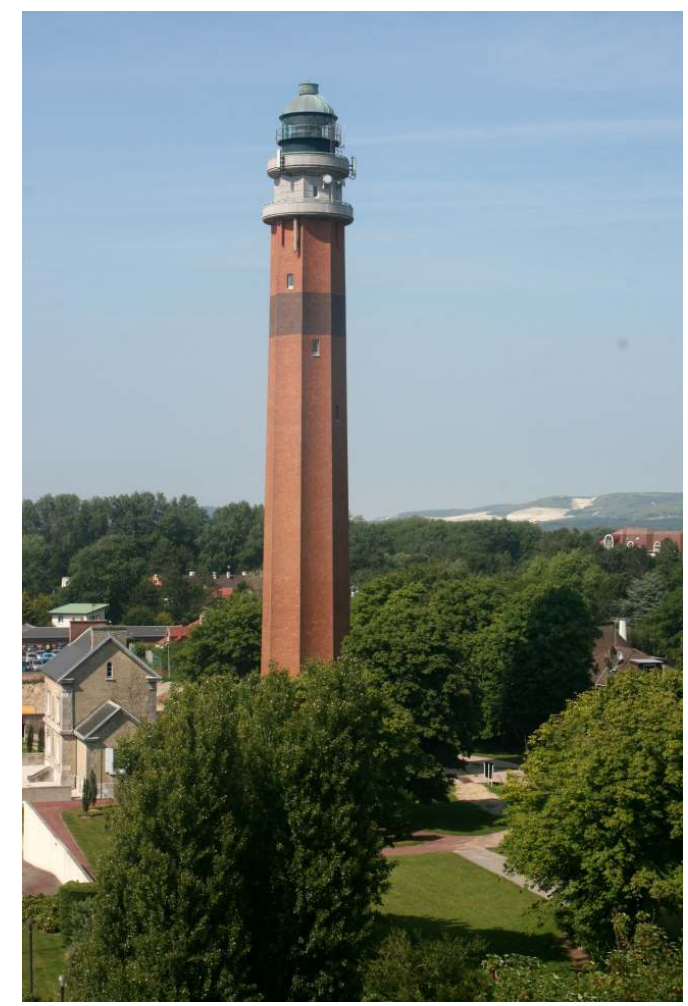

Cl. Olivier Liardet

47 L'architecte touquettois Louis Quételart a mis en valeur le phare par sa situation au centre du terrain, donnant ainsi toute son ampleur à la construction. La richesse et la diversité des matériaux employés - le soubassement en bossages de pierre ou l'utilisation des briques de différentes formes et tonalités pour le fût à l'élancement si caractéristique accentué par les parois concaves - font de ce bâtiment une incontestable réussite esthétique. Le classement au titre des monuments historiques intervenu le 19 avril 2011 lui reconnaît une place particulière dans le corpus des phares des côtes de France.

\section{NOTES}

1. Sur les problèmes de signalisation maritime, voir Jean-Christophe Fichou dir., Phares. Histoire du balisage et de l'éclairage des côtes de France, Douarnenez, 2006 et Vincent Guigueno, Au service des phares, la signalisation maritime en France XIX ${ }^{e}-X^{e}$ siècle, Rennes, 2001. 
2. Sur l'histoire de ce territoire, voir Édouard Lévêque, Histoire du Touquet et de Paris-Plage, Paris, 1996 (fac-similé de l'édition de 1905), p. 7 et suivantes.

3. Sur l'histoire des phares successifs et les problèmes de navigation voir E. Lévêque, Op. cit., p. 42-50.

4. Voir Arch. nat., $\mathrm{F}^{14} 19874$ (Phare de la Canche 1848-1951) et $\mathrm{CP} / \mathrm{F}^{14} 17523$ (plans).

5. E. Lévêque, op. cit., p. 26-41.

6. Voir E. Lévêque, op. cit., p. 53-62 et Richard Klein, Le Touquet-Paris-Plage: la côte d'Opale des années trente, Paris, 1994.

7. Guide de l'étranger. Le Touquet Paris-Plage 1959-1960, Syndicat d'Initiative du Touquet, Montreuil, 1959 , p. 3.

8. Guide de l'étranger, op. cit., p. 4-5.

9. Voir Patrick Flahaut, Les années sombres du Touquet-Paris-Plage, Le Touquet, 2005, 120 p.; Philippe Holl et Patrick Flahaut, La Seconde guerre mondiale au Touquet, Saint-Cyr-sur-Loire, 2007, $127 \mathrm{p}$.

10. Arch. nat. $\mathrm{F}^{14} 19875$, note explicative de l'ingénieur subdivisionnaire Danel, 23 octobre 1944, accompagnée d'un dessin daté 23 octobre 1944, minute de lettre du directeur du service des Phares et balises au secrétaire général de la Marine marchande, 24 octobre 1945.

11. Arch. nat., $\mathrm{F}^{14} 19875$, minute de lettre du directeur à l'ingénieur en chef de Viry, 16 octobre 1944.

12. Idem.

13. Arch. nat., $\mathrm{F}^{14}$ 19875, rapport de Danel, 23 octobre 1944.

14. Arch. nat., $\mathrm{F}^{14}$ 19875, minute de lettre du directeur à de Viry, 17 novembre 1944.

15. S'il existe peu de publications sur Quételart, certaines villas ont été publiées dans La construction moderne et L'Architecture et plusieurs ont fait l'objet d'un inventaire (voir la Base Mérimée) et d'une protection au titre des monuments historiques (Lille, D.R.A.C. Nord - Pas-deCalais, Archives de la conservation régionale des monuments historiques, abrégé en Arch. de la C.R.M.H.). Voir aussi A. Houdaille, L'Architecture dans le Nord de la France. T. II, Euvres des architectes A. Houdaille, Roger Poye, L. Quételart, A. Franche, R. Pruvost, Y. et A. Barbotin, Strasbourg, 1933; Richard Klein, Le Touquet op. cit., Paris, 1994 et Richard Klein dir., La Côte d'Opale. Architectures des années 20 et 30, Paris, 1998. Quételart a publié également plusieurs ouvrages: Louis Quételart. Quelques réalisations architecturales [à Rambouillet, à Tourcoing, du Touquet, et à l'Exposition internationale 1937], Strasbourg, 1938, 1 p. et 7 pl.; Regards sur le Touquet, s.d., 24 p.; Louis Quételart, L'Architecte, cet inconnu, préface d'Auguste Perret, Paris, 1946, 157 p.

16. D. M., "Les obsèques de M. Quételart ", Journal du Pas-de-Calais et de la Somme, mercredi 23 août 1950, p. 1.

17. Inscrite au titre des monuments historiques le $1^{\mathrm{er}}$ décembre 1997.

18. Id.

19. Id.

20. Id.

21. Sur le mouvement régionaliste voir Jean-Claude Vigato, L'architecture régionaliste. France 1890-1950, Paris, 1994.

22. La Voix du Nord, mercredi 23 août 1950, édition du littoral.

23. Arch. nat. $\mathrm{F}^{14}$ 19875, minute de lettre du directeur à Quételart, 25 novembre 1944.

24. Voir le plan annexé daté du 26 janvier 1946 (Arch. nat., $\mathrm{F}^{14}$ 19875).

25. Arch. nat., $\mathrm{F}^{14} 19875$, lettre de Quételart à Danel, 9 février 1946.

26. Arch. nat., $\mathrm{F}^{14}$ 19875, Rapport de Danel, 20 février 1946.

27. Arch. nat., $\mathrm{F}^{14} 19875$, avant-projet de reconstruction du phare annexée à la lettre de Quételart à Danel, 9 février 1946.

28. Arch. nat., $\mathrm{F}^{14} 19875$, minute de lettre du directeur au ministre des Travaux publics, 14 mars 1949 et $\mathrm{F}^{14}$ 19874, plan annexée, C. Danel, 2 mars 1949. 
29. Arch. nat., $\mathrm{F}^{14} 19875$, rapport de Danel, 20 février 1946.

30. Arch. nat., $\mathrm{F}^{14} 19875$, lettre du directeur à de Viry, 15 mai 1946.

31. Voir Lille, Arch. de la C.R.M.H., Dossier de recensement, août 2010 et Simon Texier, « Georges Tourry l'architecte anonyme, 1904-1991», Le Moniteur architecture AMC, n¹32, mars 2003, p. 66-69.

32. Arch. nat., $\mathrm{F}^{14} 19875$, lettre de l'ingénieur en chef de Viry au directeur, 18 novembre 1946.

33. Arch. nat., $\mathrm{F}^{14} 19875$, rapport de Danel au directeur, 17 février 1947 avec avis de de Viry, 19 février 1947.

34. Arch. nat., $\mathrm{F}^{14}$ 19875, minute de lettre du directeur à de Viry, 4 mars 1947.

35. Arch. nat., $\mathrm{F}^{14}$ 19875, lettre du ministre des Travaux publics et des Transports à de Viry, 13 mars 1947.

36. Arch. nat., $\mathrm{F}^{14} 19875$, lettre du ministre des Travaux publics et des Transports à de Viry, 7 mai 1948.

37. Arch. nat., $\mathrm{F}^{14}$ 19875, rapport de l'ingénieur du $2^{\mathrm{e}}$ arrondissement Briquel, 15 février 1949.

38. Arch. nat., $\mathrm{F}^{14} 19875$, lettre du ministre des Travaux publics, des transports et du tourisme à de Viry, 27 avril 1949.

39. Louis Quéterlart, "La reconstruction du phare du Touquet", La Construction moderne, septembre 1949, p. 330-331.

40. Voir les plans et élévations par faces dans les archives de l'Agence Quételart au Touquet.

41. Voir le dessin sur calque conservé dans les archives de l'Agence Quételart.

42. Arch. nat., $\mathrm{F}^{14}$ 19875, rapport de Briquel, 3 juin 1948.

43. Arch. nat., $F^{14}$ 19875, minute de lettre du directeur à de Viry, 28 juin 1948.

44. Louis Quéterlart, « La reconstruction... », Op. cit., p. 331-332.

45. Auxquels s'ajoute à l'intérieur des plaquettes de briques pour conserver l'unité d'ensemble de la cage intérieure. Voir les plans des assises et de l'élévation (s.d.) dans les archives de l'Agence Quételart.

46. Arch. nat., $\mathrm{F}^{14} 19875$, plans et élévations des logements, 15 octobre 1945 , avec modifications datées du 10 novembre 1945.

47. Arch. nat., $\mathrm{F}^{14}$ 19875, plans et élévations des logements, approuvés le 18 février 1946.

48. Arch. nat., $\mathrm{F}^{14} 19875$, plan, coupes et élévation, 15 octobre 1946.

49. Arch. nat., $\mathrm{CP} / \mathrm{F}^{14} 17523$, plan n.s., s.d.

50. Arch. nat., $\mathrm{F}^{14}$ 19875, minute de lettre du directeur à de Viry, 8 octobre 1948.

51. Arch. nat., $\mathrm{F}^{14}$ 19875, plan daté 3 février 1949 et rapport de Briquel, 15 février 1949.

52. Journal de Montreuil, dimanche 27 août 1950, p. 1.

53. Voir les analyses de Richard Klein, Le Touquet op. cit., p. 93 et sq.

54. Commande devenue possible grâce à la nouvelle organisation de la profession avec la création de l'Ordre des Architectes en 1941.

55. C'est ce qu'il rappelait dans son ouvrage Regards sur le Touquet: «L'Architecture, c'est avant tout de l'ordre. C'est un problème dont les données seront puisées dans la vie et toutes ses manifestations. Problème d'ordre par conséquent, si l'on précise que l'architecte devra, pour le résoudre avec bonheur, soumettre ou mieux, ajuster son art aux désirs de l'homme actuel dans un lieu connu, aux exigences du climat, aux conditions d'une technique vivante, à l'économie enfin « (p. 7-8).

56. Pierre sauvage, «Le grand architecte du Touquet-Paris-Plage Louis Quételart vient de mourir ", Journal du Pas-de-Calais et de la Somme, lundi 21 août 1950, p. 1.

57. Voir le dessin annoté dans les archives de l'Agence Quételart et daté du 27 avril 1956. 


\section{RÉSUMÉS}

Le site de l'estuaire de la Canche (Pas-de-Calais) a toujours été dangereux pour la navigation. Au milieu du XIX ${ }^{\mathrm{e}}$ siècle, deux phares jumeaux se dressaient sur les terrains vierges bordant le littoral de la Manche avant que ne fût créée la station balnéaire du Touquet-Paris-Plage dont on célèbre, cette année, l'érection en commune. À la fin de la seconde guerre, en quittant la ville, le 2 septembre 1944, les Allemands firent sauter les deux phares. Selon la volonté de l'administration et de la commune, la reconstruction fut confiée à l'architecte touquettois Louis Quételart (1888-1950), dont la notoriété, due à la création de villas au style très personnel, n'était plus à faire. Pour la première et la dernière fois de sa carrière, il réalisait un bâtiment public. Sa dernière œuvre fait montre d'une originalité accomplie, tant dans le choix de la mise en situation dans la ville, que par la forme du fût aux parois concaves qui donne son élancement au phare, accentué par la richesse et la diversité des matériaux employés. La mise en œuvre de la brique permet une polychromie subtile et donne un caractère régional à ce bâtiment d'une incontestable réussite esthétique. Le classement au titre des monuments historiques (19 avril 2011) lui reconnaît une place particulière dans le corpus des phares des côtes de France.

The Canche estuary in the Pas-de-Calais department of northern France has always been dangerous for navigation. In the middle of the $19^{\text {th }}$ century, two lighthouses were built at its mouth on the coast of the English Channel. In the late $19^{\text {th }}$ century, the resort town of Le Touquet Paris-Plage developed in this locale and was formally designated as a commune in 1912. At the end of the Second World War, the two lighthouses were blown up as German troupes retreated from the town (September 2, 1944). The local architect Louis Quételart (1888-1950), renowned for the highly personal style he developed for the construction of villas, was chosen by the administrators of the commune to build a new lighthouse. Completed in 1951, the lighthouse at Le Touquet is Quételart's only public commission, demonstrating the architect's originality both in its siting and in the handling of the structure. The building consists of a polygonal tower with concave facets accentuated by the rich and subtle use of materials. Polychromatic effects are achieved with brick, imparting the structure with a distinctive, regional character. Regarded as an incontestable aesthetic achievement, the lighthouse at Le Touquet was officially designated a historic monument on April 19, 2011, thus recognizing its special place in the corpus of French lighthouses.

Die Mündung der Canche erwies sich immer als eine besonders gefährliche Stelle für die Navigation. In der Mitte des 19. Jahrhunderts standen zwei Bruderleuchttürme auf unberührtem Gelände an der Küste des Ärmelkanals. So war es vor der Entstehung des Badeortes Le TouquetParis-Plage, dessen Errichtung als Gemeinde gerade dieses Jahr gefeiert wird. Am Ende des Zweiten Weltkriegs wurden beide Leuchttürme am 2. September 1944 bei dem Rückzug der deutschen Truppen gesprengt. Nach dem Willen der Stadtverwaltung wurde der ortsansässige Architekt Louis Quételart (1888-1950) mit dem Wiederaufbau beauftragt. Dieser hatte sich bisher durch den Bau von Privatvillen im originellen Stil einen besonderen Ruf erworben. Zum ersten und letzten Mal in seiner Karriere führte er also einen öffentlichen Auftrag aus. Die ganz originelle Kunstfertigkeit seines letzten Werkes lässt sich an der geschickten Einfügung des Baus in die Stadtbebauung erkennen ebenso wie an der Form der Säule mit konkaven Seitenwänden. 
Der Reichtum und die Verschiedenheit der verwendeten Materialien tragen auch zu dem hervorragenden schlanken Aussehen des Leuchtturms bei. Die subtile Polychromie der Backsteine verleiht diesem ästhetisch völlig gelungenen Gebäude (dennoch?) ein regionales Gepräge. Der Leuchtturm wurde am 19.April 2011 unter Denkmalschutz gestellt, was ihm einen besonderen Platz in dem Korpus der Leuchttürme der französischen Küsten zuweist.

159-182

\section{AUTEURS}

\section{ANNE LEFEBVRE}

Anne Lefebvre, née en 1954, est titulaire d'une licence d'histoire de l'art et d'une licence d'histoire géographie (Lille III). Chargée d'études documentaires à la direction régionale des affaires culturelles Nord - Pas-de-Calais, elle s'occupe de la protection des monuments historiques depuis 1978 et est également correspondante jardins du ministère de la Culture. Elle a mené de nombreuses campagnes thématiques de protection notamment sur le patrimoine du bassin minier en 1990-1992, puis de nouveau en 2008-2009 afin de préparer la candidature au Patrimoine mondial (Unesco) et sur les phares (campagne nationale à l'initiative du ministère de la Culture et du ministère en charge de le Mer), les deux dernières avec Olivier Liardet. Membre de la Commission supérieure des monuments historiques, section Patrimoine Industriel Scientifique et Technique (jusque 2007), elle fait également partie de la commission historique du Nord et de la commission départementale d'histoire et d'archéologie du Pas-de-Calais. Elle est l'auteur de nombreux articles et notices pour des ouvrages consacrés au patrimoine régional. Adresse électronique : anne.lefebvre@culture.gouv.fr.

Olivier Liardet, né en 1969, est chargé d'études documentaires à la conservation régionale des monuments historiques du Nord-Pas-de-Calais depuis septembre 2004, chargé de la protection au titre des monuments historiques. En collaboration avec sa collègue Anne Lefebvre, il a conduit plusieurs campagnes thématiques de protection concernant les églises de Flandre, les temples protestants, le patrimoine du bassin minier afin de préparer la candidature au Patrimoine mondial (Unesco) et les phares (campagne nationale à l'initiative du ministère de la Culture et du ministère en charge de le Mer). Dans le cadre de ce travail, il a réalisé plusieurs publications concernant le patrimoine régional. Ses autres publications portent sur l'histoire de l'architecture et le patrimoine monumental français, notamment les travaux de l'architecte Charles-Auguste Questel (1807-1888). Il s'est également intéressé à la création architecturale à Nîmes et dans le Gard dans la première moitié du XIX ${ }^{\mathrm{e}}$ siècle. Ses derniers travaux ont porté sur l'architecte Garnier dans le cadre de l'exposition Charles Garnier. Un architecte pour un empire ( Charles Garnier, voyageur éclairé ou rêveur impénitent?", Paris, ENSBA, 2010). Depuis 1999, il participe à l'élaboration de notices biographiques d'architectes et d'ingénieurs français du XIX ${ }^{\mathrm{e}}$ siècle pour l'Allgemeines Künstlerlexikon. Adresse électronique : olivier.liardet@culture.gouv.fr. 